\title{
Políticas educativas para el apoyo de trayectorias escolares en el nivel medio en América Latina: la provisión de recursos como estrategia
}

\author{
Educational policies to support school trajectories at the high \\ school in Latin America: the provision of resources as strategy \\ Pablo Daniel García \\ Universidad Nacional de Tres de Febrero, Argentina
}

Recibido: $21 / 10 / 2018$

Aceptado: 21/12/2018

\begin{abstract}
Despite the significant progress made in the extension of educational systems in Latin America and in particular with regard to the average level, the persistence of societies marked by inequality in which the Right to Education remains a promise for many children and teenagers. The object of study of the research presented in this article has been the educational policies that are developed with impact on secondary schools in Latin America that work in contexts of social vulnerability in order to improve the actual school trajectory of their students. METHOD: The research carried out was based on a qualitative logic based on the use of the Constant Comparative Method for the construction of analytical categories. The implementation of the field work involved a trip through mid-level schools in a number of Latin American countries with brief visits to 38 high school institutions in which semi-structured interviews were conducted specifically with directors and teachers in charge of projects related to the object of investigation. RESULTS: The analysis presented in the article focuses specifically on the different strategies for providing resources that are developed in educational institutions to seek to mitigate the impact of poverty on school activities. CONCLUSIONS: Outlines are outlined that allow to account for compensatory actions of social inequality to contain their impact on students' school trajectory.
\end{abstract}

KEY WORDS: high school Education; educational policies; Latin America; vulnerability; school trajectories.

\section{RESUMEN}

A pesar de los significativos avances logrados en la extensión de los sistemas educativos en América Latina y en particular en lo que respecta al nivel medio, resulta evidente la persistencia de sociedades marcadas por la desigualdad en las que el Derecho a la Educación sigue siendo una promesa para muchos niños y jóvenes. El objeto de estudio de la investigación que se presenta en este artículo han sido las políticas educativas que se desarrollan con impacto en las escuelas secundarias de América Latina que trabajan en contextos de vulnerabilidad social a fin de mejorar la trayectoria escolar real de sus estudiantes. MÉTODO: La investigación realizada se basó en una lógica cualitativa a partir del uso del Método Comparativo Constante para la construcción de categorías analíticas. La puesta en marcha del trabajo de campo realizado implicó un viaje recorriendo escuelas de nivel medio en una serie de países de América Latina con visitas breves a 38 instituciones educativas en las que específicamente se realizaron entrevistas semiestructuradas a directores y docentes a cargo de proyectos afines al objeto de investigación. RESULTADOS: El análisis que se presenta en el artículo pone el foco específicamente en las diferentes estrategias de provisión de recursos que se desarrollan en las instituciones educativas para buscar atenuar el impacto de la pobreza en las actividades escolares. CONCLUSIONES: las conclusiones esbozan algunos lineamientos que permiten dar cuenta de las acciones compensatorias de la desigualdad social para contener su impacto en la trayectoria escolar de los estudiantes.

PALABRAS CLAVE: educación secundaria; política educativa; América Latina; vulnerabilidad; trayectoria escolar.

Dirección de correspondencia:

Pablo Daniel García, Universidad Nacional de Tres de Febrero, Buenos Aires, Argentina. E-mail: pgarcia@untref.edu.ar. 


\section{Introducción}

Desde que la Declaración Universal de los Derechos Humanos (1948) fue proclamada por la Asamblea General de las Naciones Unidas se replica en una amplia cantidad de legislación en todo el mundo. Con ello, el Derecho a la Educación se instala como tema en la agenda de la mayor parte de los países y en Latinoamérica en particular, se transforma también en una de las metas por excelencia a lograr en el inicio del tercer milenio.

Con la recuperación democrática, desde la década de los ochenta, los países latinoamericanos acuerdan metas y orientaciones para la acción regional en post de la concreción del Derecho a la Educación: el Proyecto Principal de Educación para América Latina y el Caribe (1980-2000); la Convención de los Derechos del Niño (adoptada por la Asamblea de las Naciones Unidas el 20 de noviembre de 1989); el Plan de Acción de la Cumbre Mundial por la Infancia (celebrada en Nueva York en septiembre de 1990) y las Cumbres de las Américas, entre muchos otros.

Un punto clave en este recorrido lo marcó la "Conferencia Mundial de Educación para Todos", desarrollada en Jomtien (Tailandia) en marzo de 1990. Este evento representó un hito fundamental en el diálogo internacional sobre el lugar que ocupa la educación en la política de desarrollo humano. El consenso allí alcanzado dio un renovado impulso a la concreción del derecho a la educación y a la erradicación del analfabetismo.

Diez años después de la conferencia de Jomtien, los países de América evaluaron los progresos realizados en la región hacia el logro de los objetivos y metas entonces formuladas. Reunidos en Santo Domingo, en febrero del 2000, renovaron con el "Marco de Acción Regional" sus compromisos de "Educación Para Todos" para los próximos quince años. A pesar de los avances realizados, el Marco Regional dio cuenta de una serie de desafíos pendientes y dio continuidad a los esfuerzos realizados por los países en la década transcurrida para que su población alcance niveles educativos cada vez mayores. Se planteó entonces como desafío avanzar en la incorporación de jóvenes a los sistemas educativos nacionales, darles prioridad en las reformas educativas y mejorar y diversificar los programas educativos particularmente destinados a grupos excluidos y vulnerables, entre muchos otros.

Posteriormente, el Foro Mundial de la Educación de 2015, realizado en Inchon, Corea del Sur, reafirmó la visión del movimiento mundial en pro de la Educación para Todos como el acuerdo más importante en materia de educación en las últimas décadas. Se acordó un compromiso con carácter de urgencia para construir una agenda integral, ambiciosa y exigente, con el objetivo de garantizar una educación inclusiva, equitativa y de calidad y promover oportunidades de aprendizaje durante toda la vida para todos (ahora, como objetivo para el 2030).

No obstante, a pesar de los enormes avances logrados en la extensión de los sistemas educativos en Latinoamérica, resulta evidente la persistencia de sociedades marcadas por la desigualdad educativa y los núcleos de exclusión. En un escenario en donde la educación obligatoria en América Latina tiende a prolongarse, especialmente en las últimas décadas, abarcando según los países desde algún año del nivel inicial hasta la educación secundaria (Poggi, 2010), todavía queda mucho por hacer. Por ello, la situación problemática a partir de la cual se organizó esta investigación es la exclusión educativa, ya sea en su forma tradicional, manifestada en la imposibilidad de ciertos grupos sociales de acceder al sistema educativo o bien en las nuevas formas de exclusión vinculadas a la segmentación de sistema en circuitos de diferente calidad, los aprendizajes poco significativos, las repitencias múltiples que llevan al abandono y la deserción temprana.

En los últimos años se advierte en el campo de la producción de conocimientos en educación en Latinoamérica la presencia creciente de investigaciones que tienen como objeto, o como contexto institucional, la escuela secundaria. Muy probablemente este desarrollo creciente pueda explicarse, al menos en parte, por el rol central que ha tomado la escuela secundaria en la agenda de políticas educativas. En efecto, dos décadas de presencia continua de políticas de extensión de la escolarización secundaria obligatoria en la región han estimulado la preocupación por la producción de conocimientos sobre el nivel (Pinkasz, 2015).Específicamente en lo que respecta a la situación problemática a partir de la cual se organiza esta investigación resulta importante reconocer que una importante cantidad de investigaciones de los últimos años muestran una convergencia alrededor de 
la preocupación por el recorrido educativo de los jóvenes que se diferencia de estudios de décadas anteriores. Los estudios actuales no sólo se interesan por conocer la incidencia de la educación en las trayectorias sociales -y viceversa-, sino que enfocan la trayectoria escolar como producto y efecto específico del modelo de escolarización. Los estudios sobre trayectoria en un primer momento buscaron dar cuenta del tránsito por el sistema educativo a partir de las reglas propias del modelo de escolarización vigente (Baquero y otros, 2012) para luego desplazar su mirada de las estructuras educativas hacia los sujetos que las transitan. Durante los últimos años han proliferado investigaciones que ponen su foco en el estudio de programas y proyectos destinados a posibilitar la inclusión educativa en la escuela secundaria y a las traducciones y resignificaciones que producen los actores institucionales, especialmente los docentes y directivos en tanto centro de la actividad micropolítica de la escuela (Ball, 1993). Las líneas de investigación desarrolladas por Tiramonti (2004 - 2011), Terigi (2008-2009), Tenti Fanfani (2000) y Dussel (2009) entre otros, aportan datos, hipótesis y hallazgos pertinentes a esta problemática. También los estudios realizados por Kessler (2004) y Briscioli (2013) aportan desde una mirada cualitativa el análisis de las trayectorias escolares de los jóvenes que viven en contextos de desigualdad y sus problemáticas específicas. Estos estudios de referencia, sin duda, no agotan la basta la producción académica sobre estas temáticas ampliamente desarrolladas en los últimos años.

Específicamente el objeto de estudio de esta propuesta de investigación fue dar cuenta de las estrategias para mejorar las trayectorias escolares de los estudiantes que se desarrollan en las escuelas de nivel medio de Latinoamérica que trabajan en contextos de vulnerabilidad social. Este artículo en particular pone su foco en las estrategias de provisión de recursos a los estudiantes que surgen de políticas educativas que buscan compensar las desigualdades sociales.

\section{Sobre la metodología de la investigación realizada}

Siguiendo la conceptualización de Achili (1992), esta investigación se basa en una lógica cualitativa, que se vale de la inducción analítica y la búsqueda de la generación de teoría, la comprensión y la especificidad. Se trabajó siguiendo la metodología propuesta por Strauss y Corbin (1991), el denominado Método Comparativo Constante.

Se optó tomar Latinoamérica como campo para el trabajo de investigación, en principio, para tener una mirada de la diversidad de la región. La aspiración fue lograr una investigación que transite por diversidad de contextos para mostrar la complejidad del fenómeno estudiado. Se decidió abordar una selección de países de la región para estudiar una misma problemática, con los mismos instrumentos y las mismas preguntas. Esta decisión supone no limitar el análisis por aspectos institucionales, legislativos o políticos internos de los sistemas educativos de cada país en particular sino analizar una misma problemática, las intervenciones que se realizan para mejorar las trayectorias escolares de los jóvenes, en múltiples contextos de la región. Este tipo de abordaje supone la superación del denominado "nacionalismo metodológico", tal como plantea Gita Steiner-Khamsi (2012), es decir, reconocer que como resultado de los procesos de globalización, los diagnósticos sobre los desempeños de los sistemas educativos, las políticas y las propuestas de cambio (educativas, en este caso) viajan más allá de las fronteras de los países proponiendo preguntas similares para los países de la región y muy posiblemente, respuestas donde también encontrar elementos en común. En el caso particular de esta investigación, las experiencias seleccionadas se encuentran en Chile, Bolivia, Perú, Ecuador, Paraguay, Argentina y Uruguay. En relación a las unidades de análisis -las escuelas-, dado que el territorio elegido para el universo de investigación es muy vasto, la selección realizada no fue una muestra probabilística sino intencionada, es decir, con un propósito (Newton Sutter, 2006). En la presente investigación se han seleccionado escuelas de diferentes países de la región que cumplen con los siguientes requisitos: a) que sean instituciones públicas de nivel medio/secundario, b) que trabajen en contextos de vulnerabilidad social urbana o periurbana, c) que desarrollen estrategias activas para favorecer las trayectorias de los estudiantes. Esto supuso diferentes momentos para la selección de las instituciones (identificar los focos de pobreza en cada uno de los países de Latinoamérica, luego un acercamiento a información sobre la realidad educativa del lugar y 
la existencia de instituciones educativas - a partir de bibliografía académica, resultados de investigaciones previas y notas periodísticas que daban cuenta de los resultados y problemáticas educativas de las diferentes localidades visitada- y finalmente, la posibilidad de acceso al campo. Como resultado de este proceso, finalmente, quedaron seleccionadas dentro del estudio 38 instituciones educativas del nivel medio de 7 países de la región (6 instituciones de Chile, 5 de Bolivia, 5 de Perú, 6 de Ecuador, 5 de Paraguay, 7 de Argentina y 4 de Uruguay).

El abordaje de cada institución considerada dentro de la muestra de la investigación supuso una estadía breve (de 2 a 3 días en cada institución) para lograr la combinación de diversas técnicas de recolección de empiria. Las técnicas implementadas fueron principalmente entrevistas semiestructuradas a directores y/o equipos directivos de instituciones educativas centradas en las estrategias que la institución desarrolla para mejorar las trayectorias escolares. Las entrevistas semiestructuradas fueron complementadas con relatos en profundidad de experiencias de la vida institucional y lectura de documentación generada por la propia institución vinculada a acciones y/o proyectos de inclusión educativa. Así, como parte de la estrategia metodológica diseñada se optó por la triangulación de técnicas y por la combinación de fuentes de investigación (Sirvent, 2003). Fueron en total 71 entrevistas realizadas en las 38 instituciones educativas estudiadas y el trabajo de campo se ha desarrollado en diferentes momentos a lo largo de los años 2015 y 2016.

En cuanto a la técnica de análisis de la información, tal como se mencionó en párrafos anteriores, se utilizó el denominado Método Comparativo Constante. Este método ha sido desarrollado por Glaser y Strauss (1967) y retomado por Strauss y Corbin (1991). A partir del análisis de los múltiples registros se desarrolló un proceso de construcción de categorías que se exponen en los siguientes apartados.

\section{Sobre las trayectorias escolares intermitentes y heterogéneas}

El concepto de trayectoria escolar da cuenta del camino que cada estudiante debiera recorrer a través del sistema educativo. Existe una trayectoria teórica o "deseable" que no necesariamente da cuenta del recorrido educativo de cada cual (UNESCO, 2008). Varios componentes dan forma a la trayectoria escolar: en principio se define por el tiempo de ingreso (en el caso de la educación secundaria, puede ser que un joven ingrese a tiempo, ingrese tarde o no ingrese), luego puede permanecer o no y en el caso de permanecer puede avanzar un grado por año, puede repetir o abandonar temporalmente. El recorrido que cada estudiante realice por el sistema se construirá su trayectoria real (Terigi, 2009). La trayectoria real estará marcada por variables tales como el momento de ingreso, la permanencia y el momento de egreso.

A partir de la lectura de bibliografía académica sobre la temática y del análisis de las entrevistas realizadas, una primera situación que encarna una alteración en la trayectoria escolar teórica es la repitencia. Si bien las estadísticas actuales de la problemática en la región dan cuenta de un alto porcentaje de repitencia en la región, en los testimonios de los directores entrevistados aparece una notable tendencia a la baja. Otro aspecto en el que hay coincidencia en muchos de los testimonios tiene que ver con el momento del trayecto escolar donde se concentra la repitencia: el primer año. Los estudios sobre trayectorias educativas revelan que las transiciones escolares constituyen momentos delicados en los que el riesgo de discontinuidad se incrementa; la estadística educativa nacional confirma esta situación, pues en el inicio de la escuela secundaria y en el inicio de la educación superior se producen avatares característicos de las trayectorias desencauzadas (Terigi, 2014). Por lo general, la repitencia aparece asociada -al menos en el testimonio de los directores- a causas extraescolares (falta de acompañamiento de las familias, problemas socioeconómicos, inserción temprana de los jóvenes en el mundo laboral, entre otras).

Otra problemática vinculada a las trayectorias escolares reales de los estudiantes del nivel medio es el ausentismo. A partir de la influencia de factores extraescolares (lejanía con respecto a la escuela, cuidado de hermanos menores, inserción en tareas laborales de la familia, solo por mencionar algunas) muchos estudiantes comienzan a ausentarse de la escuela. El ausentismo de algún modo podría pensarse como el punto de inicio de la desligadura entre estudiante y sistema educativo. Este 
vínculo que comienza a cortarse es un claro ejemplo de cómo la exclusión educativa debe pensarse como un proceso. Un alumno no deja de asistir (en la mayor parte de los casos) definitivamente a la escuela de un día para otro. Se da un proceso de faltas constantes, llamados, profesores y otros profesionales que lo van a buscar, hasta que el estudiante finalmente, no vuelve. A partir del análisis de los testimonios, resulta interesante destacar que en los testimonios recolectados estas razones parece que tienen poco que ver con la escuela sino que están asociadas, sobre todo, a la historia personal y social de los desertores (la inserción temprana en el mercado laboral, problemas de adicciones, el desmembramiento familiar, el embarazo adolescente, entre otros) haciendo apenas alguna mención a lo escolar entre las causas.

Una situación que a veces se confunde con deserción es la denominada "movilidad escolar". En muchos casos, los entrevistados plantean la existencia de casos que abandonan la escuela pero no porque abandonen el sistema educativo sino porque sus familias toman la decisión de trasladarse a otra región dejan una escuela por otra. Varios entrevistados plantean casos de este tipo, el problema central al respecto sería analizar en qué condiciones se produce esta movilidad escolar y en qué momento del año, considerando que a partir de determinadas fechas establecidas en el calendario escolar ya no es posible suma un nuevo estudiante a una institución educativa.

Por otra parte, una situación que vinculada con el tránsito por el nivel secundario es la continuidad de estudios post-secundaria. En términos generales hay un porcentaje medio que ingresa a estudios superiores y se dividen entre estudios terciarios técnicos y las carreras universitarias tradicionales. En muchos casos, el amplio inicio del nivel superior culmina con deserciones tempranas por múltiples motivos. "Muchos quieren, pero pocos pueden" - me contaba un director en una entrevista. La necesidad de una inserción rápida en el mercado laboral, la falta de recursos para sostener la carrera y la lejanía con respecto a las instituciones del nivel superior, son algunos de los factores que aparecen mencionados en las entrevistas como limitantes para el ingreso al nivel superior de los jóvenes que viven en situación de vulnerabilidad en la región. Según los testimonios recolectados, es importante destacar que muchos estudiantes eligen trayectos de formación de carreras cortas y con una rápida inserción laboral. Por ejemplo, tecnicaturas, la docencia o incluso la formación en las fuerzas de seguridad y de defensa.

La desigualdades educativas en Latinoamérica no se expresan solamente en términos de acceso, sino también en la segregación y persistencia de circuitos educativos de menor calidad en el asociados a cuestione sociales, etnia, localización geográfica u otras situaciones. Al analizar la experiencia escolar de los jóvenes que viven en contextos de vulnerabilidad social, podemos describir sus trayectorias escolares como heterogéneas. Esta idea hacer referencia a que no existe un camino único, preformateado que todos transiten en su paso por el sistema educativo sino que aparecen dificultades del contexto y obstáculos que pone el propio sistema educativo (a través de normas, prácticas, costumbres y miradas estigmatizadoras, por ejemplo) que los conducen por caminos distintos al formal. Se trata de estudiantes que llegan al nivel secundario acumulando repitencia, deben distribuir el tiempo de estudio con trabajo informal en el marco de sus familias o para terceros, los barrios donde viven están lejos de las escuelas por lo cual solo llegar ya les resulta difícil en contextos donde falta infraestructura, adquirir materiales y libros para cumplir sus tareas escolares resulta una problemática dada la escasez de recursos familiares, solo por mencionar algunas de las situaciones a las que estos jóvenes y adolescentes están expuestos. Ingresan, desertan, repiten, vuelven, se ausentan, aprueban alguna materia, buscan otras oportunidades, vuelven a desertar, vuelven a reingresar. Los itinerarios escolares de estos jóvenes son realmente diversos e imprevisibles. Si bien en la mayoría de los países de la región se vienen desarrollando políticas públicas orientadas al incremento de los años de obligatoriedad escolar y entendiendo a la educación como un derecho de los niños, adolescentes y jóvenes, el trabajo pendiente todavía es mucho.

Cuando el formato escolar prestablecido, sus normas rígidas y tradiciones se encuentra con contextos cargados de vulnerabilidad aparece la desigualdad escolar y los caminos en el tránsito por el sistema educativo se diversifican y complejizan. Ingreso tardío, acumulación de repitencias, rezago, ausentismo, deserción, movilidad son fenómenos que marcan las trayectorias escolares de los jóvenes y adolescentes (en este caso, particularmente los de sectores vulnerables) y los llevan por múltiples 
posibles caminos. Si bien estas situaciones que suponen alteraciones en las trayectorias no están per se vinculadas a un sector social en particular, el contexto de vulnerabilidad aporta un condicionamiento negativo que no resulta posible omitir. La situación de vulnerabilidad de partida de ellos (y de sus familias) sin dudas condiciona (pero no determina) su paso por la escolaridad. Es en el encuentro entre estas situaciones sociales se encuentran con los supuestos y necesidades inflexibles del sistema educativo donde las situaciones de desigualdad se generan.

\section{Las intervenciones: algunas categorías para pensar las estrategias de inclusión}

Considerando las problemáticas esbozadas de manera preliminar en los párrafos anteriores, los diferentes estados latinoamericanos han desarrollado una amplia diversidad de políticas buscando tener un impacto positivo en las trayectorias educativas de los jóvenes y adolescentes de la región, y en particular de los que provienen de sectores más vulnerables. Estas estrategias muchas veces suponen generar nuevas oportunidades para los estudiantes con mayor riesgo educativo y se plasman en las intervenciones más diversas. Las instituciones educativas, por su parte, no solo se apropian de estas políticas en su llegada a las aulas sino que a la vez generan sus propias acciones. Al recorrer las escuelas de Latinoamérica queda claro que la cuestión del acceso, la retención y la finalización del ciclo de enseñanza media es uno de los temas prioritarios hoy en día en la gestión escolar.

Un aspecto central del análisis del material empírico recopilado durante el trabajo de campo fue la construcción de categorías analíticas a partir de las estrategias para la mejora de las trayectorias escolares identificadas. El amplio abanico de estrategias puesto en marcha para lograr la inclusión educativa plena de los jóvenes y adolescentes podría clasificarse con múltiples criterios. Una clasificación posible de estrategias podría realizarse a partir de considerar la forma de intervención que suponen, otro podría ser el momento de la trayectoria educativa donde se busca tener un impacto y finalmente otro podría ser el origen de la creación de la estrategia.

En relación al tipo de intervención que suponen las estrategias con impacto en la trayectoria educativa relevadas, podría arriesgarse una clasificación que distinga varias áreas de trabajo, que se combinan de diferentes modos en cada institución. En principio se postula la existencia de cinco tipos de intervenciones:

- Estrategias de provisión de recursos, a partir de brindar a los estudiantes algún tipo de bien del cual carecen o podrían carecer (por ejemplo, libros o vianda escolar)

- Estrategias de innovación en la gestión educativa, a partir de pensar nuevos espacios institucionales que permitan formas innovadoras de planificación, gestión y tomada de decisiones institucionales.

- Estrategias basadas en el acompañamiento a los estudiantes, básicamente para contener el desarrollo de sus trayectorias escolares y generar espacios de escucha y participación.

- Estrategias enfocadas en lo pedagógico, que suponen formas alternativas de dictado de las clases, formas innovadoras de trabajo docente o de evaluación de los estudiantes, entre otras posibilidades.

- Acciones espontáneas del equipo docente (frente a emergentes ocasiones, sin planificación previa y dispersas).

Específicamente en los párrafos que siguen del presente artículo se analizan en mayor profundidad las denominadas estrategias de provisión de recursos.

\section{La provisión de recursos como estrategia para enfrentar la desigualdad educativa}

A partir del trabajo de campo realizado, las estrategias intervención para la mejora de las trayectorias escolares basadas en la provisión de recursos a los estudiantes pueden dividirse al menos en 4 subcategorías: estrategias de provisión de recursos económicos, estrategias de provisión de 
recursos escolares, estrategias de provisión de recursos alimenticios y estrategias de provisión de recursos complementarios

La provisión de recursos económicos a las familias se desarrolla a partir de diferentes categorías de intervenciones. En varios de los países de la región aparecen programas que se basan en la asignación de becas focalizadas en la población de mayor condición de vulnerabilidad. Con diversas modalidades, la provisión de becas se desarrolla en Chile, Perú, Bolivia y Uruguay. En el caso de Chile, las becas ocupan un lugar fundamental en la estrategia de abordaje a la vulnerabilidad particularmente, a través de varias becas que atienden a poblaciones específicas. El entramado complejo de becas que organiza la JUNAEB en Chile, con varios tipos de beca, de acuerdo a una focalización específica que se organiza desde el nivel central, supone una provisión o transferencia de recursos directa hacia sectores desfavorecidos. Este tipo de intervenciones pueden considerarse inversión social ya que su objetivo es lograr un impacto sobre las condiciones de vida de las familias receptoras (Martínez Nogueira, 2006). Resultan de particular interés las becas denominadas BARE. Estas becas tienen como objetivo estimular y apoyar a los y las estudiantes que presentan alto riesgo de abandonar el sistema escolar, para que logren finalizar con éxito su enseñanza media. En el listado de condiciones para la postulación a estas becas se establece: el acceso a través de una presentar factores de riesgo de deserción (sobreedad respecto al nivel cursado, baja asistencia el año inmediatamente anterior en los registros de Ministerio de Educación), condición de paternidad o maternidad, condición de embarazo o padres de hijos en gestación, estar registrado como infractor en algún programa de protección del SENAME (Servicio Nacional de Menores) dependiente del Ministerio de Justicia o presentar una discapacidad acreditada en el Registro Nacional de Discapacidad (JUNAEB, 2014).

También puede incluirse dentro de esta subcategoría de intervenciones (estrategias de provisión de recursos económicos) el caso de Bolivia, donde existe un estímulo con aspiración universal, el Bono Juancito Pinto. Este bono lo cobran todos los estudiantes que asisten a determinados grados de la educación pública (los seis grados del nivel primario y los dos primeros cursos de secundaria de las unidades educativas fiscales). El programa se encuentra vigente desde el año 2006 a partir del Decreto Supremo 28.899 y tiene como metas a largo plazo contribuir a incrementar la matrícula escolar y reducir la deserción, así como disminuir la trasmisión intergeneracional de la pobreza. El pago es anual y el monto total del bono es de 200 bolivianos (aproximadamente unos 30 dólares). En las entrevistas realizadas en Bolivia en general aparece una mirada negativa hacia el bono ("es poco", "se les da a todos", "debería focalizar en quienes tienen buenas calificaciones").

Resulta importante destacar además que Perú y Argentina tienen programas de asignación de recursos monetarios para las poblaciones más vulnerables que se organizan por fuera del sistema educativo. En el caso de Perú, el "Programa Juntos", un programa de transferencia de recursos la población "más pobre". En el caso argentino, la "Asignación Universal por Hijo", supone un pago mensual que se otorga a personas desocupadas, a quienes trabajan empleados en negro o a quienes ganan menos del salario mínimo, vital y móvil, por cada hijo menor de 18 años o hijo discapacitado. Ambas propuestas, no obstante, poseen una contraprestación educativa dado que para participar de dichos beneficios los beneficiarios deben demostrar la continuidad escolar de sus hijos.

Los programas de transferencias condicionadas se han difundido considerablemente durante las dos últimas décadas en la región a través de gobiernos de distintas corrientes políticas. Algunos analistas afirman que la condicionalidad de este tipo de propuestas puede tener un objetivo político, además de su objetivo explícito o instrumental (asegurar la asistencia a la escuela o la concurrencia a cierta cantidad de controles pediátricos por ejemplo), pues en el imaginario social justifica el gasto público, que de otra forma se vería como una medida populista o como un incentivo para dejar de trabajar (Pritchett, 2012). Otros análisis, también críticos, consideran que la condicionalidad de los programas de transferencias está asociada a ideologías liberales que relacionan la pobreza con capacidades, elecciones y causas individuales, antes que con causas estructurales, por lo que la condicionalidad sumada a la transferencia proporciona al individuo un incentivo para que tome la responsabilidad de salir de su situación de pobreza (Lund y otros, 2008). 
Un caso radicalmente diferente de estrategias de provisión de recursos económicos es el de Uruguay con el Programa Compromiso Educativo. El objetivo del programa, desarrollado por el Consejo Directivo Central de la Administración Nacional de Educación Pública (ANEP), es contribuir a que adolescentes y jóvenes accedan, permanezcan y potencien sus trayectorias en el sistema educativo público y logren finalizar así completar la Educación Media Superior. Las becas de estudio conforman uno de los componentes de este programa, y constituyen un apoyo económico que busca contribuir al acceso y mantenimiento de los jóvenes en la educación media, en los casos en los que el aspecto económico un problema. No todos los jóvenes que participan del programa acceden a la beca. Quienes la desean la solicita y se evalúa su situación. La beca es de $\$ 8000$ anuales (aprox. USD275) en 2017. El Programa Compromiso Educativo, no obstante, se trata de un caso de estrategia mixta dado que si bien incluye una entrega de dinero específico a un grupo de estudiantes con ciertos requisitos, también su puesta en marcha supone otros componentes que le dan una complejidad mayor. El Programa Compromiso Educativo además de la beca promueve la firma de un "Acuerdo Educativo", que deben suscribir las autoridades de la institución, un adulto referente y el estudiante donde se especifican las metas y acciones concretas que las partes se comprometen a realizar para la mejora de la trayectoria escolar. Cada acuerdo responde a una situación particular y es único. Por otra parte, el programa incluye otro componente que es el "Espacios de Referencia entre Pares" que es un ámbito abiertos a todas/os las/os estudiantes del Centro Educativo del que participan estudiantes de nivel terciario y universitario, que acompañan a los estudiantes del liceo. La puesta en marcha de estos tres componentes supone la articulación de un entramado complejo de diversas instituciones públicas: el Consejo Directivo Central, y los Consejos de Formación en Educación, de Educación Secundaria y de Educación Técnico Profesional; el Instituto del Niño y el Adolescente del Uruguay además del Ministerio de Educación y Cultura y el Ministerio de Desarrollo Social. Partiendo de una realidad que se reconoce compleja, multidimensional y multicausal, Compromiso Educativo supone el trabajo de múltiples instituciones para, a partir de distintos instrumentos, acompañar las trayectorias escolares de los estudiantes, potenciándolas y promoviendo su continuidad. Según los datos disponibles, en 2014 el programa estuvo presente en 95 centros educativos de todo Uruguay y a lo largo de sus cuatro años de ejecución, se han firmado alrededor de 19.500 "Acuerdos Educativos", se han entregado más de 18.000 becas de estudio, y han participado más de 1.700 estudiantes universitarios o de nivel terciario como tutores, generando un espacio de acompañamiento y seguimiento a los más de 25.000 estudiantes de escuela media superior que han participado (según datos disponibles en el programa hasta el 2015). Este tipo de intervenciones va más allá de la mera provisión de recursos y busca intervenir en la mejora de la experiencia escolar de los estudiantes que transitan por el programa.

Otro modo de intervención desarrollado por los países de la región se basa en la distribución de recursos para el desarrollo de las tareas escolares en el inicio de cada ciclo lectivo. Son intervenciones que -al igual que las anteriores- buscan garantizar los recursos mínimos necesarios para que la escolarización pueda realizarse. Se trata de propuestas con aspiración universal (es decir que buscan cubrir a la totalidad de la población que asiste a la educación pública, sin importar su condición económica) aunque particularmente tiene un impacto muy positivo en los sectores más vulnerables. En los caso de Chile, Paraguay, Ecuador y Argentina, una de las estrategias vigentes es la provisión a cada estudiante de instituciones de gestión pública de útiles para el desarrollo de sus actividades escolares. En el caso de Paraguay, como signo distintivo, se destaca que los "kit escolares" son diferentes para cada año de escolaridad dado que se arman en función de las actividades que se prevén realizar de acuerdo a los contenidos establecidos para dicho año. En Argentina y Ecuador, la distribución de útiles se complementa con la dotación de libros escolares a estudiantes y bibliotecas.

Otra forma de manifestación de esta estrategia es la provisión de uniformes escolares. Una experiencia interesante al respecto es la que se desarrolla en Ecuador a través del Programa "Hilando el Desarrollo" a través del cual se encarga a cooperativas e industrias locales la confección de los uniformes escolares. Este programa comenzó a ser implementado en el año 2007 y es ejecutado por el Ministerio de Educación y el Servicio Ecuatoriano de Capacitación Profesional. Por ejemplo, en la Unidad Educativa Ambato, visitada en el marco del trabajo de campo, los estudiantes usan un 
uniforme escolar basado en los colores rojo y azul que son los colores tradicionales de la comunidad originaria de la zona. A través de "Hilando el Desarrollo" se busca generar modelo de desarrollo socioeconómico, local y solidario que articule el sector productivo artesanal, con la inclusión de los pequeños artesanos del país para reactivar las economías locales. Por ello el programa incluye un componente de capacitación a los artesanos locales para la producción de uniformes escolares de manera gratuita para los niños y niñas de establecimientos fiscales de las zonas rurales de todas las provincias del país.

Finalmente, otra forma de manifestación de esta estrategia es a través de la provisión de tecnología. En este sentido, fue vanguardia en la región el Plan Ceibal desarrollado a partir del Decreto $\mathrm{N}^{\circ} 144 / 07$ en Uruguay para acortar la brecha de acceso al mundo digital en la niñez y la juventud. A diez años de su creación, el Plan Ceibal atiende al 85\% de los estudiantes del sistema educativo uruguayo: el 100\% de los estudiantes de educación pública de entre 6 y 15 años y sus docentes, como también los estudiantes de institutos no públicos de zonas carenciadas. Cada estudiante recibe un dispositivo en primero y cuarto de Primaria, y en primero de Enseñanza Media. Además, la red de conexión generada por Ceibal conecta más de 1.500 centros educativos en todo el país (Ceibal, 2017). En Argentina, el Programa Conectar Igualdad fue creado en abril de 2010 a través del Decreto $N^{\circ} 459 / 10$, para recuperar y valorizar la escuela pública y reducir las brechas digitales, educativas y sociales en el país. Conectar Igualdad desde 2010 distribuyó notebooks a estudiantes y docentes de las escuelas secundarias, de educación especial y de los institutos de formación docente de gestión estatal de Argentina, además entre sus objetivos creacionales estuvo la búsqueda de la mejora de los procesos de enseñanza y aprendizaje a través de la modificación de las formas de trabajo en el aula y en la escuela a partir del uso de las TIC. La implementación de estos programas de provisión de tecnología de forma masiva desarrollada tanto en Uruguay como en Argentina busca favorecer la utilización de las TICs en las instituciones educativas. Ahora bien, la mera presencia de las notebooks no es suficiente para mejorar la calidad educativa. En este complejo proceso de integración curricular de la tecnología, hay muchos agentes implicados y muchos factores que determinan su calidad.

Otra forma de concreción de las estrategias de provisión de recursos se vincula con la alimentación escolar. La alimentación escolar juega un rol clave en la nutrición de los niños y jóvenes de sectores vulnerables, y es una condición esencial para una vida saludable y para el aprendizaje. Por el amplio alcance del sistema educativo, la escuela resulta un espacio estratégico para que el Estado llegue a todos los sectores de la población y asegure las condiciones básicas nutrición y sanidad, además de transmitir hábitos alimenticios y de higiene (Britos y otros, 2003). En todos los países de la región donde se ha realizado el trabajo de campo se desarrolla una estrategia de provisión de recursos alimenticios durante la jornada escolar. Más allá de este punto en común, resulta interesante destacar las variaciones que se observan en la implementación de esta estrategia.

En primer lugar, existen importantes diferencias en relación al responsable del desarrollo de los Programas de Alimentación Escolar (PAE). Por ejemplo, existen modelos de organización de los PAE que son responsabilidad del gobierno central (Estado Nacional, a través del Ministerio de Educación o de otro ministerio). Este es el caso de Perú y Ecuador, por ejemplo. Otros países como Argentina o Uruguay tienen modelos de gestión de la alimentación escolar basados en las jurisdicciones (gobernaciones). En Bolivia, por ejemplo, la responsabilidad es de los gobiernos locales (municipales). Resulta importante considerar este punto al momento de analizar el servicio brindado: municipios insolventes o diferencias entre las posibilidades económicas de las jurisdicciones impiden pensar en un servicio universal, sino que por el contrario, prevalecen las quejas por su insuficiencia.

En segundo lugar, es posible realizar una diferenciación entre aquellos PAE que mantienen una lógica de focalización y aquellos que se sostienen en una matriz universalista. En relación a la focalización, el caso más extremo es el de Chile: cada estudiante de un liceo es analizado de acuerdo a su condición de vulnerabilidad para determinar si le corresponde o no recibir alimentación. De tal modo, en un mismo liceo, puede ocurrir que haya estudiantes que reciben beneficios de alimentación y otros que no. Otro modo de focalización es el de Ecuador, donde el foco se pone en instituciones educativas que se elige beneficiar o no. Por ejemplo, desde el 2013 se dispuso la provisión de almuerzo 
escolar en las Unidades Educativas del Milenio, nuevas instituciones que se construyen en los distritos de mayor vulnerabilidad. En el caso de Perú, el PAE Qali Warma tiene diferente tipo de cobertura de acuerdo a las condiciones de vulnerabilidad de una comunidad: entrega 2 raciones (desayuno y almuerzo) a estudiantes que asisten a escuelas ubicadas en distritos de mayor pobreza y una ración (desayuno) a estudiantes que asisten a escuelas ubicadas en distritos de menor pobreza.

En tercer lugar, otra distinción puede realizarse entre el origen de los fondos que sostienen los PAE. En el caso de Argentina, Paraguay y Uruguay, se trata de fondos públicos. En los casos de Perú y Bolivia se menciona la contribución, además de fondos públicos, de fondos privados y de ONG. Un caso particular es el de Ecuador, donde se da, en algunas instituciones, un sistema mixto donde el Estado contribuye con los recursos pero son las familias quienes se hacen cargo de su preparación. La rectora de la Unidad Educativa Olón narraba durante la entrevista:

Se les da la colación escolar, que consiste en, bueno a muchas instituciones les llega la leche líquida de distintos sabores para poder dar a los chicos y a las chicas con galleta... acá nos llega la papilla para la preparación de la colada, allí también los padres nos colaboran... los docentes los reparten por lista entre los estudiantes, los padres lo preparan y lo traen el día que les toca... la traen ya preparada y la sirven a los chicos. Se prepara con agua, con leche, con todo lo que los padres quieran ponerle, canela... (Entrevista Rectora, Unidad Educativa Olón, Ecuador).

Finalmente, una última distinción entre los PAE puede establecerse entre los que simplemente se proponen proveer alimentos a los estudiantes, con una lógica de tipo distributiva y aquellos que le suman componentes pedagógicos. En este sentido, puede destacarse el caso de Uruguay donde comedor escolar se organiza considerando varias funciones: la alimentación, la socialización y la educación de los estudiantes. Los directivos escolares participan de este ámbito y aprovechan los intercambios que allí se dan con los estudiantes.

En general, los comedores escolares están presentes en la región desde los años ochenta. El aumento de la pobreza y de la brecha de desigualdad a fines del milenio, exigió ampliar la oferta de alimentación escolar. Pese a la posterior recuperación macroeconómica de algunos países, la pobreza estructural y los problemas nutricionales persistentes mantuvieron la necesidad de la alimentación escolar (Díaz Langou y otros, 2014). Si bien en principio los servicios de alimentación escolar surgieron para las escuelas primarias, los cambios recientes en la obligatoriedad escolar y la ampliación de la jornada escolar llevaron a su extensión al nivel secundario. Pese a que, desde sus orígenes la gestión de los servicios alimentarios se fue institucionalizando, investigaciones recientes sobre la temática afirman que restan realizar en la región importantes esfuerzos para garantizar las condiciones para ofrecer comidas de calidad en todas las escuelas de la región (Díaz Langou y otros, 2014). En muchos de los países aún son infrecuentes las leyes específicas o las partidas presupuestarias para ciertas dimensiones para algunas de las dimensiones que supone la puesta en marcha de un comedor escolar tales como el personal a cargo de preparar y servir las comidas en las escuelas, la infraestructura, la educación nutricional o el monitoreo y la evaluación del servicio brindado (FAO, 2013).

Las intervenciones para garantizar la alimentación de los estudiantes es posible pensarlas también como estrategias que buscan acotar una de las formas de desafiliación -en términos de Castel (1995)- más duras: el hambre. Sin dudas, el contexto de desigualdad estructural y de pobreza de la región demanda la continuidad de estos tipos de intervenciones para que otras, más ligadas a lo pedagógico, sean posibles.

Una última forma de manifestación de estrategias de provisión de recursos es la provisión de recursos complementarios a la vida escolar. Este subtipo de estrategias incluye una amplia variedad de acciones de acuerdo a las políticas vigentes en cada país. Resulta de interés pensarlas como parte del andamio que se genera a las trayectorias escolares sin estar directamente vinculadas a lo pedagógico o a lo escolar.

Un primer grupo de propuestas se agrupa en torno al transporte para el acceso a las instituciones educativas. Se considera una dimensión importante para aliviar la economía familiar y así garantizar el acceso a la educción a costo nulo o muy bajo. Al respecto Ecuador, Argentina, Paraguay y Uruguay hay organizado sistemas de descuento para el transporte público, por lo general, 
gestionados a nivel municipal. En Ecuador además hay un sistema de autobuses escolares organizados en algunos municipios para movilizar especialmente a la población de sectores más vulnerables hacia las unidades educativas (particularmente para garantizar el acceso a las Unidades Educativas del Milenio). Perú además ha organizado un sistema de distribución de bicicletas a través del Programa Rutas Solidarias que distribuye entre estudiantes de escuela primaria y media en zonas de mayor pobreza del país. Junto con la bicicleta se distribuyen cascos de seguridad, chalecos con cintas reflectantes y cajas con juegos de herramientas y repuestos básicos.

Finalmente, dos iniciativas para destacar dentro de este grupo de recursos complementarios que se otorgan a los estudiantes para estimular su permanencia en las instituciones educativas, se refieren a dos proyectos desarrollados por el Cantón de Guayaquil, en Ecuador.

- El Programa Bachiller Digital, que entrega a todos los graduados del nivel medio una Tablet (lo paradójico es que el Programa se llama Bachiller Digital pero el dispositivo lo entregan cuando ya ha terminado de cursar el nivel). Este proyecto forma parte del Programa "Más Educación", con el que se busca fortalecer la calidad de educación en el cantón e incentivar a la ciudadanía a aprender y desarrollarse.

- El Programa Jóvenes Ejemplares premia la excelencia de los jóvenes de escuelas de gestión pública (fiscales y fiscomisionales -administradas por congregaciones o por el clero-) a través de la asignación de múltiples recursos a los estudiantes con los mejores promedios que se gradúan en cada institución. El premio principal es la construcción de una casa para el graduado con mejor promedio del colegio.

En términos generales, en todos los países se sostienen estrategias de provisión de recursos a los estudiantes (de manera más focalizada o de tipo universal). Detrás de ellas persiste el supuesto de la necesidad de sostener políticas redistributivas dada la situación de pobreza estructural y la persistencia de las situaciones de desigualdad que caracterizan la región. La provisión de recursos a los estudiantes busca (en los distintos países y de diferentes modos) el acceso a recursos económicos, recursos escolares (útiles y uniformes, entre otros), recursos alimentarios (desayunos, colaciones o almuerzos, de acuerdo a los diferente casos) y otros recursos complementarios (como por ejemplo, transporte para acceder a las instituciones educativas).

Uno de los interrogantes que orientó la investigación se refería a quiénes son los actores protagónicos de las estrategias identificadas para la mejora de las trayectorias escolares. Los estados centrales tienen un alto grado de participación en la construcción de intervenciones para la mejora de las trayectorias escolares. La mayor parte de los programas relevados tiene una ejecución diseñada y llevada a cabo por las instancias centrales del sistema educativo. Estas intervenciones por lo general toman la forma de programas o proyectos (dado que se supone que son más ágiles que otras formas de gestión de política pública). Por otra parte, estas acciones han recuperado su intención de "universalidad" frente a las políticas focalizadas propias de los años 90. La recuperación de la idea de "universalidad" de las propuestas y las intervenciones ha llegado al nivel secundario junto con la sanción de su obligatoriedad y por ende, la responsabilidad del Estado de generar condiciones de acceso, permanencia y promoción para toda la población en edad escolar. La noción misma de "Derecho a la Educación" y la recuperación en la legislación de la centralidad del Estado en la garantía de este derecho dan lugar al desarrollo de estrategias que busquen cierta transversalidad y cobertura de la totalidad del sistema. Se da además un desplazamiento de la preocupación por la equidad a una mayor preocupación por la inclusión y la "igualdad". Una variación dentro de estas intervenciones sistémicas son las que han denominado "transversales", que asumen el principio de la discriminación positiva en la aplicación de medidas generales. Es decir, ante la sanción de políticas con cobertura universal, se privilegia la intervención en primer lugar sobre determinadas instituciones priorizadas que cumplen con ciertas características y una vez que se realiza la intervención sobre ellas, se avanza sobre el resto. 
En relación a las estrategias de provisión directa de recursos, en general, en ellas es el nivel central de la administración del sistema quien tiene el predominio (organiza y ejecuta la distribución).

\section{Conclusiones}

Si bien los estados de Latinoamérica han hecho grandes esfuerzos a través de las políticas públicas para reducir la pobreza, los esfuerzos todavía no alcanzan. Latinoamérica sigue siendo una región fragmentada entre quienes viven con enormes privilegios y quienes padecen numerosas exclusiones. Millones de niños y jóvenes viven en situación de extrema pobreza, con sus derechos negados, sin cubrir sus necesidades básicas y con un incierto que amenaza su desarrollo y pone en riesgo su trayectoria escolar si el sistema educativo no le da un espacio acorde, ya que son muchos los que fracasan, repiten o abandonan la escuela. Esta investigación -aun en curso- busca hacer dar respuestas esta problemática.

La plena inclusión educativa es una responsabilidad indelegable del Estado. Y no se resuelve simplemente con una ampliación de la red escolar. La expansión es simplemente una de las formas de luchar contra la exclusión educativa. Pero no es la única ni es suficiente. Es necesario avanzar en el estudio de las condiciones pedagógicas e institucionales que hagan posible, a los jóvenes y adolescentes en situación de vulnerabilidad escolar, no solamente ingresar al sistema educativo, sino permanecer en él y lograr los aprendizajes que les permitan el ejercicio de una ciudadanía plena.

Hay una realidad ineludible: la desigualdad social y educativa no tiene que ver, solamente, con los sujetos de la pobreza. Para pensar la desigualdad debemos también pensar en los procesos de exclusión y sus cómplices. Y en este punto, el sistema educativo no resulta ajeno. Esta investigación busca aportar una mirada no ingenua, crítica, atenta sobre las acciones que realizan las escuelas en pos de la inclusión educativa. Luego de años de reformas educativas a lo largo de toda la región, las políticas de inclusión no logran alcanzar la proclamada "educación para todos". Aquí se abren algunos interrogantes sobre los que avanzará la presente investigación ¿Qué éxitos y fracasos pueden evaluarse en las múltiples formas de intervención ensayadas para lograr el cumplimiento pleno del derecho a la educación para los jóvenes y adolescentes de Latinoamérica? En definitiva, las estrategias que desarrollan las escuelas de Latinoamérica que dicen trabajar por la inclusión educativa ¿Incluyen o reproducen lógicas excluyentes?

\section{Referencias bibliográficas}

Ball, S. (1993). La Micropolítica de la Escuela. Hacia una teoría de la organización escolar. Barcelona: Paidós.

Baquero, R., Terigi F., Toscano, A., Briscioli, B., Burlatti, S. (2012). La obligatoriedad de la escuela secundaria. Variaciones en los regímenes académicos. Revista Espacios en Blanco, Serie Indagaciones (22), 77-112.

Briscioli, B. (2013). Tendencias y puntos críticos en las trayectorias escolares de estudiantes de Escuelas de Reingreso de la Ciudad de Buenos Aires. (Tesis doctoral para optar por el grado de Doctora en Pedagogía de la Facultad de Ciencias de la Educación de la Universidad Nacional de Entre Ríos).

Britos, S.; O’Donnell, A.; Ugalde, V. y Clacheo, R. (2003). Programas Alimentarios en Argentina. Buenos Aires: CESNI.

Castel, R. (1995). La metamorfosis de la cuestión social. Una crónica del salariado. Buenos Aires: Paidós.

CEIBAL (2017). Plan Ceibal, 10 años. 2007-1017. Montevideo, GCPC. Disponible en: http://www.ceibal.edu.uy/storage/app/media/documentos/ceibal-10-2.pdf

Díaz Langou, G.; Bezem, P.; Aulicino, C.; Cano Belén, E. y Sánchez, B. (2014). Los modelos de gestión de los servicios de comedores escolares en Argentina. Documento de Trabajo N ${ }^{\circ} 121$. Buenos Aires: CIPPEC. 
Dussel, I. (2009). ¿Qué lugar tiene la escuela media en la producción y reproducción de la desigualdad? Elementos para el debate. Revista de Politica Educativa, 1(1), 68-90.

FAO. (2013). Alimentación escolar y las posibilidades de compra directa de la agricultura familiar: estudio de casos de ocho países. Proyecto GCP/RLA/180/BRA. Brasil: FAO

Glaser, B. y Strauss A. (1967). The discovery of grounded theory. Chicago: Aldine Publishing Company.

JUNAEB (2014). Informe de Seguimiento de Programas Sociales. Beca de Apoyo a la Retención Escolar (Programa de Educación Media). Publicación online. Consultado en: http://www.programassociales.cl/pdf/2015/PRG2015_3_513.pdf

Kessler, G. (2004). Sociología del delito amateur. Buenos Aires: Paidós.

Lund, F., Noble, M., Barnes, H. y Wright, G. (2008) Is there a rational for conditional cash transfer for children in South Africa? Working Paper No 53, July 2008.

Martínez Nogueira, R. (2006). Desafíos estratégicos en la implementación de programa sociales. Provisión, participación y coordinación. En J.C. Cortazar Velarde (ed.), Entre el diseño y la evaluación: el papel crucial de la implementación de los programas sociales. Washington: BID.

Newton Sutter, W. (2006). Introduction to educational research. Thousand Oaks: Sage Publications.

Pinkasz, D. -Comp.- (2015). La investigación sobre educación secundaria en la Argentina en la última década. Buenos Aires: FLACSO Argentina.

Poggi, M. (2010). Una radiografía de los sistemas educativos de América Latina. Desafíos para las políticas educativas. Pensamiento Iberoamericano, 7, 3-25.

Pritchett, L. (2012) Impact Evaluation and Political Economy: What Does the "Conditional". En Conditional Cash Transfers" Accomplish?, Global Development: Views from the Center, Center for Global Development, January 12.

Sirvent, M.T. (2003). El proceso de investigación. Ficha I. Cátedra de Investigación y Estadística Educacional I. Facultad de Filosofía y Letras. UBA. OPFyL

Steiner-Khamsi, G. (2012). Lo político y lo económico de la comparación. Revista Latinoamericana de Educación Comparada, 3(3), 9- 22.

Strauss, A. y Corbin, J. (1991). Basics of qualitative research. London - New Delhi: Sage.

Tenti Fanfani E. (2000). Culturas juveniles y cultura escolar. Documento presentado al seminario "Escola Jovem: un novo olhar sobre o ensino médio", organizado por el Ministerio da Educaçao. Secretaria de Educaçao Média e Tecnológica. Coordenaçao-Geral de Ensino Médio, Brasilia.

Terigi, F. (2008) Los cambios en el formato de la escuela secundaria argentina: Por qué son necesario, porqué son tan difíciles. Revista Propuesta Educativa, 29(1), 63-71.

Terigi, F. (2009). Las trayectorias escolares, del problema individual al desafio de politica educativa. Buenos Aires: Ministerio de Educación de la Nación.

Terigi, F. (2014). Trayectorias escolares e inclusión educativa: del enfoque individual al desafío para las políticas educativas. En A. Marchesi; R. Blanco y L. Hernández (coords.), Avances y desafíos de la educación inclusiva en Iberoamérica (pp. 71-81). Madrid: Organización de Estados Iberoamericanos/ Fundación MAPFRE. Colección Metas Educativas 2021.

Tiramonti, G -Comp- (2004). La trama de la desigualdad educativa. Mutaciones recientes en la escuela media. Buenos Aires: Manantial.

Tiramonti, G. (2007). Nuevos formatos escolares para promover la inclusión educativa. Un estudio de caso: la experiencia argentina. Buenos Aires: FLACSO - CealCI.

Tiramonti, G. (2011). (Dir) Variaciones sobre la forma escolar. Limites y posibilidades de la escuela media. Rosario: FLACSO - Homo Sapiens

UNESCO (2008): Un enfoque de la EDUCACIÓN PARA TODOS basado en los derechos humanos. Marco para hacer realidad el derecho de los niños a la educación y los derechos en la educación. Nueva York, UNESCO/UNICEF. 


\section{Para citar este artículo}

García, P.D. (2018). Políticas educativas para el apoyo de trayectorias escolares en el nivel medio en América Latina: la provisión de recursos como estrategia. Revista Fuentes, 20(2), 37-50. [Fecha de consulta: dd/mm/aa].

doi: http://dx.doi.org/10.12795/revistafuentes.2018.v20.i2.03 